\title{
Cholesterol efflux is LXRa isoform-dependent in human macrophages
}

\author{
A Zhi Sha Ma ${ }^{1,2}$, Zhi Yuan Song ${ }^{1 *+}$ and Qian Zhang ${ }^{1 *+}$
}

\begin{abstract}
Background: The nuclear receptor liver X receptor (LXR) has two isoforms: LXRa and LXRß. LXR activation promotes cholesterol efflux in macrophages, but the relative importance of each LXR isoform in mediating cholesterol efflux remains elusive.

Methods: We evaluated the ability of different doses of LXRs agonist T0901317 to affect cholesterol efflux in human macrophages and its relationship with mRNA and protein levels of several well-characterized proteins involved in cholesterol efflux, including $A B C A 1, A B C G 1, S R-B I, L X R \beta$ and $L X R a$, using quantitative real-time PCR, Western blotting, and siRNA techniques.

Results: Here we show that LXRa rather than LXRß sustains baseline cholesterol efflux in human blood-derived macrophages. Treatment of human macrophages with a non-isoform-specific LXR agonist T0901317 substantially increased HDL- and apoA-I-mediated cholesterol efflux, which was associated with increased mRNA and protein expression levels of ABCA1, ABCG1, SR-BI, LXRa and LXRß. The siRNA- mediated silencing of LXRa, but not LXR $\beta$ significantly reduced the protein levels of $A B C A 1, A B C G 1$, and SR-Bl as wellas HDL- and ApoA1-mediated cholesterol in human macrophages.
\end{abstract}

Conclusions: These findings imply that LXRa- rather than LXRß- specific agonists may promote reverse cholesterol transport in humans.

Keywords: Reverse cholesterol transport, Liver X receptor, siRNA, ABC transporter, Atherosclerosis

\section{Background}

Atherosclerosis is characterized by the presence of cholesterol-laden macrophages (foam cells) within the arterial wall $[1,2]$. The formation of foam cells is a result of disrupted balance between cholesterol uptake and efflux in macrophages. Macrophage cholesterol efflux is predominantly mediated by ATP-binding cassette $(\mathrm{ABC})$ transporters $\mathrm{A} 1$ (ABCA1), ABCG1, and scavenger receptor class B type I (SR-BI) [3]. It is the initial step of reverse cholesterol transport (RCT), a process that removes excess cholesterol from peripheral tissues/cells including macrophages to circulating high density lipoproteins (HDLs) for fecal disposal via the hepatobiliary route [4]. Liver X receptors (LXRs) are nuclear receptors that function as cholesterol sensors and regulate transcription of a set of

\footnotetext{
*Correspondence: zysong2010@126.com; zhangq931@126.com

${ }^{\dagger}$ Equal contributors

'Department of Cardiology, Southwest Hospital, The Third Military Medical University, Chongqing, China

Full list of author information is available at the end of the article
}

genes associated with cholesterol absorption, transport, efflux and excretion, thus playing a pivotal role in cholesterol homeostasis in vivo [5]. There are two LXR isoforms, LXR $\alpha$ and LXR $\beta$. Each of them forms a heterodimer with a retinoid X receptor (RXR) to activate target gene expression by 3 binding to LXR response elements (LXREs) located in the promoter 3 regions of the target genes [6]. LXR $\alpha$ and LXR $\beta$ are similar in structure, ligand-binding domains (LBDs), and DNA-binding domains (DBDs), but their nuclear retention and localization as well as functions display some differences [7]. The two isoforms may have evolved from one ancestor. Pufferfish has only one $\mathrm{LXR} \alpha$, which is more closely related to mammalian LXR genes by sequence similarity, yet the pattern of tissue expression more closely resembles mammalian LXR $\beta$ genes in its ubiquity of expression [8]. The sequence data suggests that the two LXR isoforms are likely duplicated from a single ancestor LXR gene, and this duplication is concurrent with the evolution of mammals [9]. In 
mammals, LXR $\alpha$ is abundantly expressed in the liver, adipose tissue, small intestine, kidneys and macrophages, whereas the LXR $\beta$ isoform is ubiquitously expressed [10]. T0901317 is a general agonist for both LXR isoforms [11]. It has been shown that the activation of LXRs by T0901317 facilitates cholesterol efflux in macrophages and inhibits atherosclerosis in animal models $[12,13]$. However, the relative importance of each LXR isoform in mediating cholesterol efflux in human macrophages remains elusive. In this study, we demonstrate that the baseline cholesterol efflux in human blood-derived macrophages depends on LXR $\alpha$, but not LXR $\beta$, implying a potential role of LXR $\alpha$ specific activation in enhancing reverse cholesterol transport in humans.

\section{Methods}

\section{Materials}

LXRs agonist T0901317 was from Sigma, USA. Total RNA extraction reagent RNAiso Plus, PrimeScript RT reagent kit, and SYBR-Green PCR kit were obtained from Takara (Japan). Immunoblot reagents were purchased from the Beyotime Institute of Biotechnology (China). LXR $\alpha$ siRNA and LXR $\beta$ siRNA were synthesized by Shanghai Genechem (Shanghai, China). All other chemicals were of the best grade available from commercial sources.

\section{Cell culture}

Human peripheral blood monocytes were isolated from blood samples of 3 healthy volunteers using Ficoll/Hypaque gradient centrifugation. The pooled monocytes were incubated in DMEM supplemented with $10 \%$ autologous serum for 10 days so that they would differentiate into macrophages. Written informed consent was obtained from all subjects for participation in the study, and the protocol was approved by the ethics committee of Southwest Hospital.

\section{Cellular cholesterol efflux assays}

Human macrophages were cultured as indicated above. Macrophages were then labeled with $\left[{ }^{3} \mathrm{H}\right]$-cholesterol $(0.3 \mu \mathrm{Ci} / \mathrm{mL})$ in serum-free DMEM containing $50 \mu \mathrm{g} / \mathrm{mL}$ ox-LDL and $0.2 \%$ bovine serum albumin (BSA) for $24 \mathrm{~h}$. The cells were washed twice with phosphate-buffered saline (PBS) and incubated in $25 \mathrm{~mL}$ of DMEM containing $0.2 \%$ BSA with or without LXRs agonist T0901317 at 5 or $10 \mu \mathrm{mol} / \mathrm{L}$ for $48 \mathrm{~h}$. The media were then replaced with DMEM containing $0.2 \%$ BSA in the presence of lipid-free apoA-I $(10 \mu \mathrm{g} / \mathrm{mL})$ or HDL $(50 \mu \mathrm{g} / \mathrm{mL})$ for $24 \mathrm{~h}$. Efflux media were collected and centrifuged to remove floating cells. Monolayer cells were washed twice with PBS, and cellular lipids were extracted using isopropanol. The radioactivity in media and cell-associated $\left[{ }^{3} \mathrm{H}\right]$-cholesterol was then measured using a liquid scintillation counter. The percent efflux was calculated with the following equation: [total medium counts/ (total cellular counts + total medium counts) $] \times 100 \%$.

\section{RNA isolation and quantitative realtime PCR analysis}

Total RNAs were extracted using RNAiso Plus reagents according to manufacturer's instructions. PCR primers were synthesized by Shanghai Sangon (Shanghai, China) and the primer sequences used were as follows: ABCA1: forward primer: 5-AAG CCA AGC ATC TTC AGT TC-3', reverse primer: 5'-CCA TAC AGC AAG AGC AGA AGG-3'; ABCG1: forward primer: 5'ATA CAG GGG AAA GGT CTC CAA T-3', reverse primer: 5'-CCC CCG AGG TCT CTC TTA TAG T-3'; SR-BI: forward primer: 5'-AGG GAT AGG GTT GGA GTC AGC-3', reverse primer: 5'CGT TGT AAT GGA AGC CAG AGG-3'; LXR $\alpha$ : forward primer: 5'-AGG CCG GTG CTG AGT ATG TC-3', reverse primer: 5'-GGG CTC CAT AAA GTC ACC AA -3'; LXR $\beta$ : forward primer: 5'-TGT CGT GTG CTC AGT ATG TG-3, reverse primer: 5'-AGC CGC CAT ATA GTC ACT GT-3'; and GAPDH: forward primer: 5-AGG CCG GTG CTG AGT ATG TC-3, reverse primer: 5'-TGC CTG CTT CAC CAC CTT CT-3'. Real-time quantitative PCR was performed with $\mathrm{SYBR}^{\oplus}$ Premix Ex Taq ${ }^{\mathrm{Tm}}$ II on a Bio-Rad LightCycler with an iQ3.1 realtime PCR system. Melt curve analysis of all real-time PCR products was used to produce a single DNA duplex. Quantitative measurements were obtained using the $\Delta \Delta \mathrm{Ct}$ method. GAPDH was used as an invariant internal control.

\section{Western blot analysis}

Cells were harvested and protein extracts prepared in accordance with the manufacturer's instructions. Immunoblot analysis [12\% SDS-PAGE; $30 \mu$ g proteins per lane] was then performed using rabbit anti-ABCA1, anti-ABCG1, anti-SRBI, anti- LXR $\alpha$, anti-LXR $\beta$ and anti-GAPDH antibodies (Abcam, USA). Proteins were visualized using Enhanced Chemiluminescence.

\section{Screening for effective LXR siRNA fragments}

SiRNAs specific for human LXR $\alpha$ and LXR $\beta$ and the nonsilencing (control) siRNAs were synthesized by Shanghai Genechem (Shanghai, China). Human macrophages $(1 \times$ $10^{\wedge} 6$ cells/well) were transfected with each siRNA using Lipofectamine2000 (Invitrogen). Following $48 \mathrm{~h}$ transfection, the second siRNA fragment (5'-AAC TCA ATG ATG CTG AGT T-3', LXR $\alpha$-siRNA) targeting LXR $\alpha$ suppressed LXR $\alpha$ expression by $70 \%$, and the third siRNA fragment (5'-ATG TCA CTG ATT CTG AGT AA-3', LXR $\beta$ - siRNA) targeting LXR $\beta$ suppressed LXR $\beta$ expression by $75 \%$ according to realtime PCR results.

\section{LXR siRNA transfection and Western blot analysis}

Human macrophages were grown in culture flasks at a density of $1 \times 10^{\wedge} 7 / \mathrm{mL}$ for $12 \mathrm{~h}$, washed twice with PBS, 
and then incubated in DMEM containing 10\% autologous human serum. The non-targeting control siRNA, LXR $\alpha$ siRNA and LXR $\beta$ siRNA were added to the culture flasks separately, and cultured for $96 \mathrm{~h}$. Cells were then harvested and protein extracts prepared in accordance with the manufacturer's instructions. The proteins were then subjected to immunoblot analysis [12\% SDS-PAGE; $60 \mu \mathrm{g}$ protein per lane] using a rabbit anti-ABCA1, anti-ABCG1, anti-SR-BI, anti- LXR $\alpha$, anti-LXR $\beta$ or anti-GAPDH (Abcam, USA)-specific antibody. Proteins were visualized using Enhanced Chemiluminescence reagents.

\section{Cholesterol efflux assays}

Human macrophages were cultured as indicated above. Human macrophages were then labeled with $\left[{ }^{3} \mathrm{H}\right]$-cholesterol $(0.3 \mu \mathrm{Ci} / \mathrm{mL})$ in serum-free DMEM containing $50 \mu \mathrm{g} / \mathrm{mL}$ ox-LDL and $0.2 \%$ BSA for $24 \mathrm{~h}$. The cells were washed twice with PBS, cultured in DMEM containing $0.2 \%$ BSA, then treated with siRNAs as described above. Seventy-two hours post-siRNA treatment, the media were replaced with DMEM containing $0.2 \%$ BSA and lipid-free apoA-I $(10 \mu \mathrm{g} / \mathrm{mL})$ or HDL $(50 \mu \mathrm{g} / \mathrm{mL})$ for cholesterol efflux assays as described above.

\section{Statistical analysis}

Data are expressed as Mean \pm Standard Error of the Mean (SEM). Results were analyzed using one-way ANOVA with SPSS 13.0 software. $\mathrm{P}<0.05$ was considered statistically significant.

\section{Results}

LXRs agonist T0901317 enhances cholesterol efflux in human macrophages

To determine if LXRs agonist T0901317 promotes cholesterol efflux in human blood-derived macrophages, we measured HDL- and apoAI-mediated cholesterol efflux in these macrophages. At 5 and $10 \mu \mathrm{mol} / \mathrm{L}, \mathrm{T} 0901317 \mathrm{sig}-$ nificantly increased HDL-mediated cholesterol efflux by $36 \%$ (from $30.3 \%$ to $41.2 \%$ ) and $50 \%$ (from $30.3 \%$ to $45.6 \%$ ) (Figure 1A) as well as apoA-I-mediated cholesterol efflux by $115 \% 9$ (from $2.6 \%$ to $5.6 \%$ ) and $165 \%$ (from $2.6 \%$ to $6.9 \%$ ) (Figure 1B), respectively.

\section{LXRs agonist T0901317 increases mRNA and protein} expression levels of genes involved in cholesterol efflux in human macrophages

ABCA1 and ABCG1 are LXR target genes critically involved in cholesterol efflux [14]. As expected, LXRs agonist T0901317 treatment increased mRNA levels of ABCA1 and ABCG1 by $550 \%$ and $605 \%$ respectively at $5 \mu \mathrm{mol} / \mathrm{L}$, and $780 \%$ and $945 \%$ respectively at $10 \mu \mathrm{mol} / \mathrm{L}$ in human macrophages (Figure 2A). The mRNA levels of SR-BI, LXR $\alpha$ and LXR $\beta$ were also elevated significantly by $255 \%$, $560 \%$, and $365 \%$, respectively, at $5 \mu \mathrm{mol} / \mathrm{L}$, and by $470 \%$, $895 \%$ and $515 \%$, respectively, at $10 \mu \mathrm{mol} / \mathrm{L}$. Similar changes were found for the protein levels of these genes (Figure 2B, 2C and 2D). In human macrophages, T0901317 treatment increased the protein expression levels of ABCA1, ABCG1, SR-B1, LXR $\alpha$ and LXR $\beta$ by $295 \%, 309 \%, 355 \%, 550 \%$, and $485 \%$, respectively, at $5 \mu \mathrm{mol} / \mathrm{L}$, and by $560 \%, 490 \%, 520 \%$, $740 \%$, and $690 \%$, respectively.

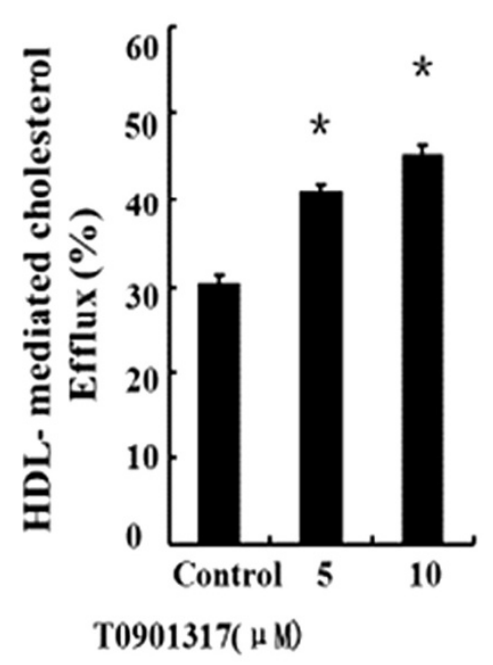

A

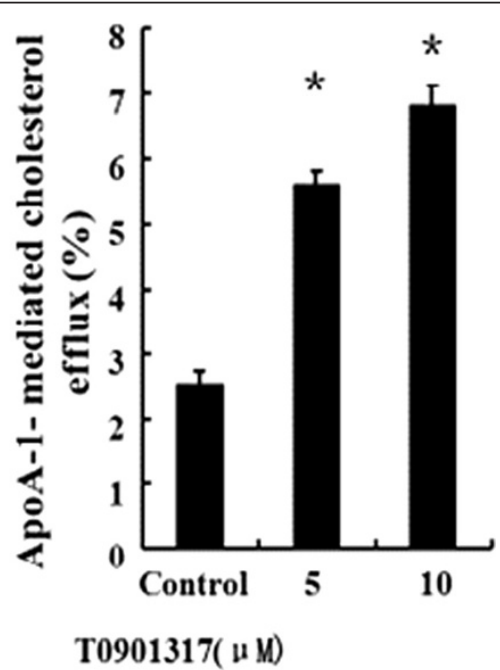

B

Figure 1 LXRs agonist T0901317 enhances cholesterol efflux from human macrophages. Following $24 \mathrm{~h}$ of labeling with $\left.{ }^{3} \mathrm{H}\right]$-cholesterol, human macrophages were treated with T0901317 or the vehicle for $48 \mathrm{~h}$. Cells were then washed with PBS, and assayed for apoA-I- (A) or HDL- (B) mediated cholesterol efflux as described under the Methods. Data in each group were obtained from triplicate flasks. Data are presented as Mean $\pm \mathrm{SEM},{ }^{*} \mathrm{P}<0.05$ (vs control). 


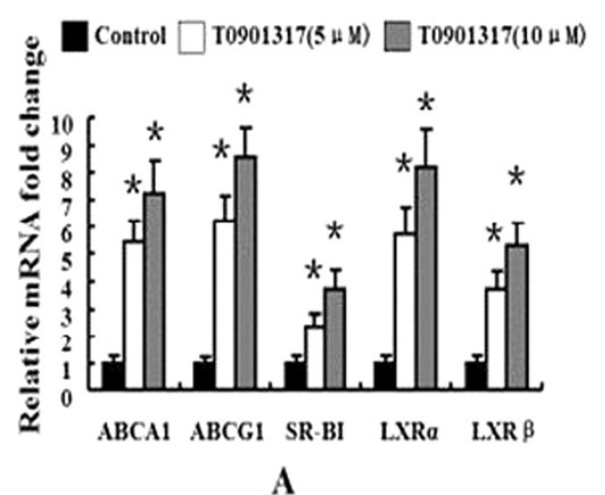

$\mathrm{T} 0901317(\mu \mathrm{M}) \quad 0 \quad 5 \quad 10$

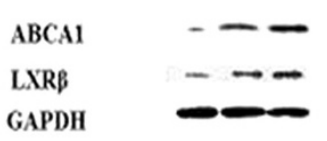

C
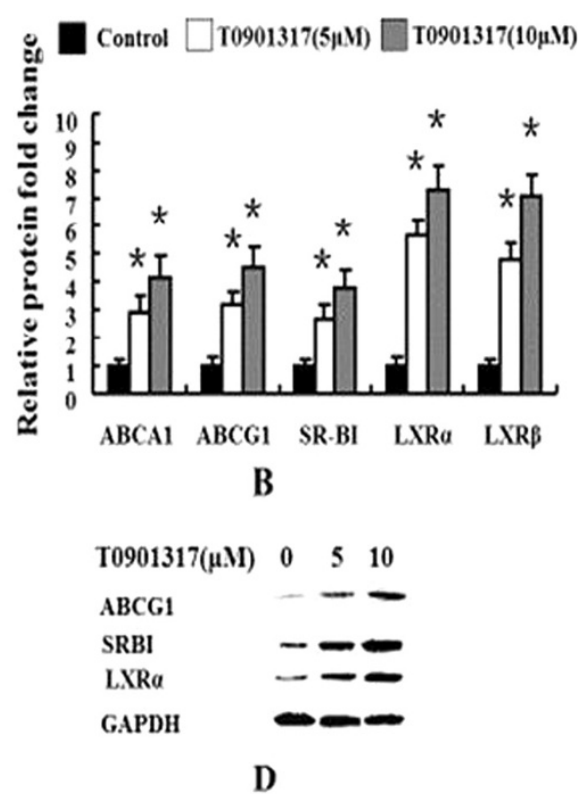

Figure 2 LXR agonist T901317 increases expression of genes related to cholesterol efflux. Human macrophages were treated without or with LXRs agonist T0901317 at $5 \mu \mathrm{mol} / \mathrm{L}$ or $10 \mu \mathrm{mol} / \mathrm{L}$ for $24 \mathrm{~h}$, and ABCA1, ABCG1, SR-BI, LXRa and LXRß mRNA expression levels were measured by real-time quantitative PCR (A). Human macrophages were treated without or with LXRs agonist T0901317 at $5 \mu \mathrm{mol} / \mathrm{L}$ or $10 \mu \mathrm{mol} / \mathrm{L}$ for $48 \mathrm{~h}$, and $A B C A 1, A B C G 1, S R-B I, L X R a$ and $L X R \beta$ protein expression levels were measured by immunoblotting $(\mathbf{B}, \mathbf{C}$ and $\mathbf{D})$. Similar results were obtained in three independent experiments. Data are presented as Mean \pm SEM, ${ }^{*} \mathrm{P}<0.05$ (vs control).

\section{$L X R a$ rather than $L X R \beta$ sustains the baseline levels of $A B C A 1, A B C G 1$ and SR-BI expression in human macrophages}

To determine the relative importance of each LXR isoform in maintaining baseline levels of ABCA1, ABCG1 and SR-BI expression in human macrophages, we independently silenced each LXR isoform in these cells. Silencing of LXR $\alpha$ ( $79 \%$ efficiency) in human macrophages reduced the protein expression levels of ABCA1, ABCG1, and SR-BI to $21.7 \%, 24.4 \%$, and $28.2 \%$ of each non-targetin control siRNA group, respectively, without affecting the protein expression of LXR $\beta$. However, when LXR $\beta$ expression was silenced by $\sim 78 \%$ in human macrophages, the protein expression levels of ABCA1, ABCG1, and SR-BI were not affected (Figure $3 \mathrm{~A}, 3 \mathrm{~B}$ and $3 \mathrm{C}$ ).

\section{LXRa rather than LXR $\beta$ sustains the baseline cholesterol efflux in human macrophages}

To determine the relative contribution of each LXR isoform to baseline levels of cholesterol efflux in human macrophages, we independently silenced each LXR isoform by the siRNA approach and measured HDL- and apoAI-mediated cholesterol efflux in these cells (Figure 4A and $4 \mathrm{~B})$. LXR $\alpha$ siRNA treatment significantly reduced HDL-, and apoA-I- dependent cholesterol efflux by $\sim 48 \%$ (from $33 \%$ to $17.2 \%$ ) and $\sim 69 \%$ (from $3.1 \%$ to $0.95 \%$ ), respectively, in human macrophages. However, these effects were not observed in human macrophages treated with LXR $\beta$ siRNA.

\section{Discussion}

The major finding of this study is that LXR $\alpha$ rather than $\mathrm{LXR} \beta$ expression is required for sustaining the baseline protein expression levels of ABCA1, ABCG1 and SR-BI, as well as HDL- and apoA-I- mediated cholesterol efflux in human blood-derived macrophages. There has been increasing interest in developing LXR agonists to promote cholesterol efflux and subsequent reverse cholesterol transport. LXR $\alpha$ activation was shown to promote cholesterol efflux and reverse cholesterol transport [15], making LXRs as attractive drug targets. However, simultaneous activation of both LXR $\alpha$ and LXR $\beta$ by a synthetic agonist T0901317 induces hepatic steatosis [16], an unwanted side effect. It was speculated that compounds activating LXR $\alpha$ in a tissue-specific manner or specifically targeting LXR $\alpha$ might be useful. Results from subsequent animal studies are in agreement with this speculation $[17,18]$. Our results demonstrate that the role of LXR $\alpha$ in mediating cholesterol efflux in human macrophages is minimal. While developing LXR agonists for use in humans, we may need to focus on agonists specifically targeting LXR $\alpha$ isoform. Our data are consistent with previous findings showing that LXRs agonist T0901317 increases expression of ABCA1 and ABCG1 to mediate cholesterol efflux in macrophages 


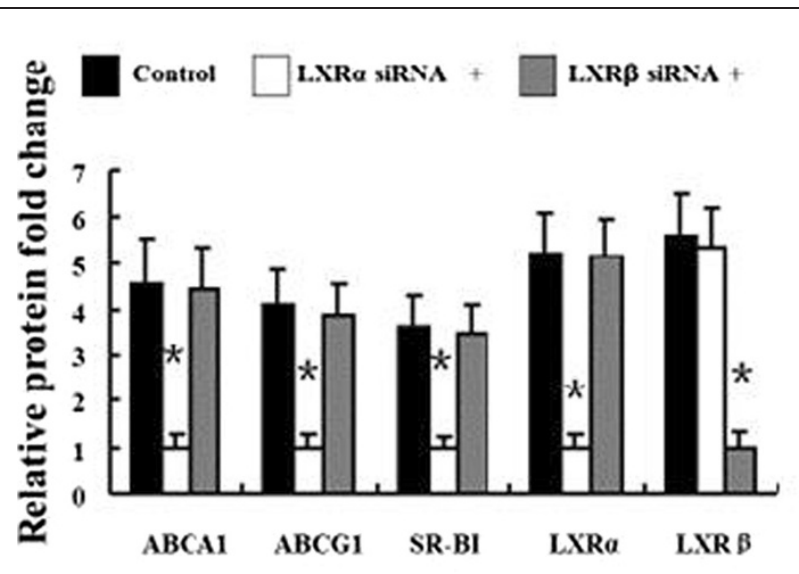

A

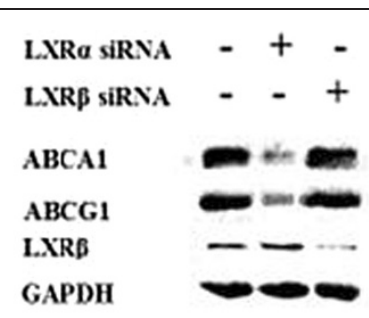

B

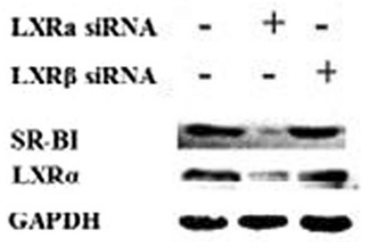

C

Figure 3 The baseline protein expression levels of ABCA1, ABCG1 and SR-BI depend on LXRa, but not LXR $\beta$ in human macrophages. Human macrophages were transfected with non-targeting control, LXRa siRNA, or LXRß siRNA, and then incubated for 96 h. ABCA1, ABCG1, SR-Bl, $L X R a$ and $L X R \beta$ protein expression levels were measured by immunoblotting (A, B and C). Similar results were obtained in three independent experiments. Data are presented as Mean $\pm \mathrm{SEM},{ }^{*} \mathrm{P}<0.05$ (vs non-targeting control).

[13]. SR-BI mediates bidirectional cholesterol flux [19]. Here we found that T0901317 increases SR-BI mRNA and protein levels in human blood-derived macrophages. It is currently unclear how LXRs agonist T0901317 regulates SR-BI mRNA and protein expression. Nonetheless, increased SR-BI expression has the potential to increase the bidirectional cholesterol transport, thus unlikely contributing to increased cholesterol efflux in T0901317-treated human macrophages. The relative importance of each LXR isoform in sustaining baseline expression levels of ABCA1 and ABCG1 as well as cholesterol efflux was unclear in human macrophages. In this study, we found that LXR $\alpha$ isoform is essential and LXR $\beta$ is dispensable for maintaining baseline ABCA1 and ABCG1 expression and cholesterol efflux in human blood-derived macrophages. During the preparation of this manuscript, a study with

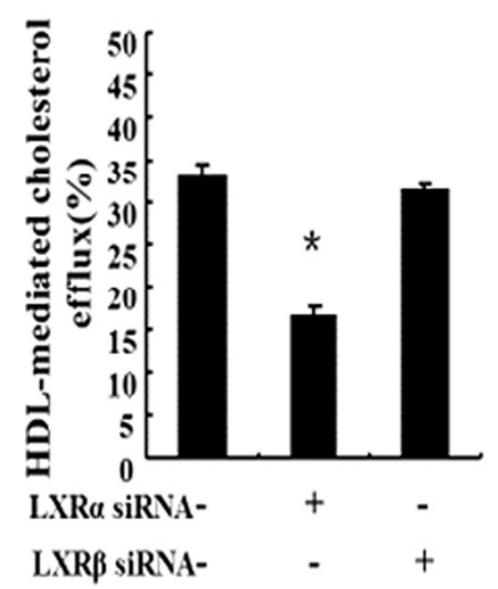

A

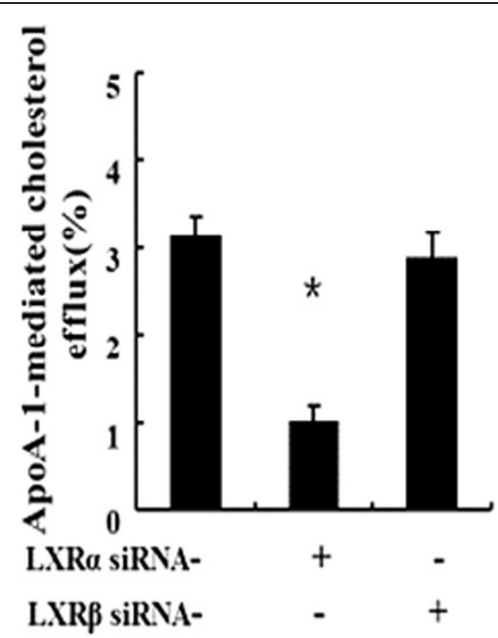

B

Figure 4 The baseline levels of cholesterol efflux depend on LXRa, but not LXR $\beta$. in human macrophages. After labeled with [ $\left.{ }^{3} \mathrm{H}\right]-\mathrm{cholesterol}$ for $24 \mathrm{~h}$, human macrophages were transfected with non-targeting control, LXRa, or LXRB siRNA, and incubated in DMEM containing $0.2 \%$ BSA for $72 \mathrm{~h}$. The media were then replaced with DMEM containing 0.2\% BSA in the presence of apoA-I (10 $\mu \mathrm{g} / \mathrm{mL})$ (A) or HDL (50 $\mu \mathrm{g} / \mathrm{mL})$ (B) for $24 \mathrm{~h}$, a ssayed for cholesterol efflux in triplicate as described under the Methods. Data are presented as Mean \pm SEM, *P $<0.05$ (vs non-targeting control). 
similar results was published [20], demonstrating that our finding is a reproducible phenomenon in human macrophages. The two studies collectively warrant detailed molecular studies of this reproducible observation in the future.

\section{Conclusion}

In conclusions, LXR $\alpha$ rather than LXR $\beta$ plays a predominant role in mediating cholesterol efflux in human macrophages.

\section{Competing interests}

The authors declare that they have no competing interests.

\section{Authors' contributions}

AZSM designed the experiments and wrote the manuscript; AZSM carried out cell culture, the molecular genetic studies, the immunoassays and inhibition using LXR siRNA. ZYS participated in study design andcoordination and helped to draft the manuscript. All authors read and approved the final manuscript. QZ performed cellular cholesterol efflux experiments and statistical analyses.

\section{Acknowledgements}

This work was supported by the Natural Scienc Foundation of China, fund No. 8110219.

\section{Author details}

${ }^{1}$ Department of Cardiology, Southwest Hospital, The Third Military Medical University, Chongqing, China. ${ }^{2}$ Department of Cardiology, The fifth hospital of Chinese PLA, Yinchuan, China.

Received: 21 February 2014 Accepted: 26 June 2014

Published: 4 July 2014

\section{References}

1. Lusis AJ: Atherosclerosis. Nature 2000, 407:233-241.

2. Bobryshev $\mathrm{Y}$ : Monocyte recruitment and foam cell formation in atherosclerosis. Micron 2006, 37:208-222.

3. Escolà-Gil JC, Rotllan N, Julve J, Blanco-Vaca F: In vivo macrophage-specific RCT and antioxidant and antiinflammatory HDL activity measurements: New tools for predicting HDL atheroprotection. Atherosclerosis 2009, 206:321-327.

4. Lewis GF, Rader DJ: New insights into the regulation of HDL metabolism and reverse cholesterol transport. Circ Res 2005, 96:1221-1232.

5. Willy PJ, Umesono K, Ong ES, Evans RM, Heyman RA, Mangelsdorf DJ: LXR, a nuclear receptor that defines a distinct retinoid response pathway. Genes Dev 1995, 9:1033-1045.

6. Repa JJ, Mangelsdorf DJ: The role of orphan nuclear receptors in the regulation of cholesterol homeostasis. Annu Rev Cell Dev Biol 2000, 16:459-481.

7. Kugimiya A, Takagi J, Uesugi M: Role of LXRs in control of lipogenesis. Tanpakushitsu Kakusan Koso 2007, 52(13 Suppl):1814-1815.

8. Maglich JM, Caravella JA, Lambert MH, Willson TM, Moore JT, Ramamurthy L: The first completed genome sequence froma teleost fish (Fugu rubripes) adds significant diversity to the nuclear receptor superfamily. Nucleic Acids Res 2003, 31:4051-4058.

9. Reschly EJ, Ai N, Welsh WJ, Ekins S, Hagey LR, Krasowski MD: Ligand specificity and evolution of liver $\mathrm{X}$ receptors. J Steroid Biochem Mol Biol 2008, 110:83-94.

10. Prüfer K, Boudreaux J: Nuclear localization of liver $\mathrm{X}$ receptor alpha and beta is differentially regulated. J Cell Biochem 2007, 100:69-85.

11. Zelcer $\mathrm{N}$, Tontonoz $\mathrm{P}$ : Liver $\mathrm{X}$ receptors as integrators of metabolic and inflammatory signaling. J Clin Invest 2006, 116(3):607-614.

12. Terasaka N, Hiroshima A, Koieyama T, Ubukata N, Morikawa Y, Nakai D, Inaba T: T-0901317, a synthetic liver $X$ receptor ligand, inhibits development of atherosclerosis in LDL receptor-deficient mice. FEBS Lett 2003, 536:6-11.
13. Peng D, Hiipakka RA, Reardon CA, Getz GS, Liao S: Differential anti-atherosclerotic effects in the innominate artery and aortic sinus by the liver $x$ receptor agonist T0901317. Atherosclerosis 2009, 203(1):59-66.

14. Tang SL, Chen WJ, Yin K, Zhao GJ, Mo ZC, Lv YC, Tan, Ouyang XP, Yu XH, Kuang $H J$, Jiang ZS, Fu YC, Tang CK: PAPP-A negatively regulates $A B C A 1, A B C G 1$ and SR-B1 expression by inhibiting LXR through the IGF-I-mediated signaling pathway. Atherosclerosis 2012, 222:344-354.

15. Tontonoz $P$, Margesdorf DJ: Liver $X$ receptor signaling pathway in cardiovascular disease. Mol Endocrinol 2003, 17:985-993.

16. Gao M, Le B, Yongjie M, Dexi L: Concurrent Activation of Liver X Receptor and Peroxisome Proliferator-Activated Receptor Alpha Exacerbates Hepatic Steatosis in High Fat Diet-Induced Obese Mice. PLoS One 2013, 8(6):e65641.

17. Janowski BA, Willy PJ, Devi TR, Falck JR, Mangelsdorf DJ: An oxysterol signaling pathway by the nuclear receptor LXR alpha. Nature 1996, 383:728-731.

18. Alberti $S$, Schuster $G$, Parini P, Feltkamp D, Diczfalusy U, Rudling M, Angelin B, Björkhem I, Pettersson S, Gustafsson JA: Hepatic cholesterol metabolism and resistance to dietary cholesterol in LXRß-deficient mice. J Clin Invest 2001, 107:565-573.

19. Ji A, Meyer JM, Cai L, Akinmusire A, de Beer MC, Webb NR, van der Westhuyzen DR: Scavenger receptor SR-BI in macrophage lipid metabolism. Atherosclerosis 2011, 217(1):106-112.

20. Ishibashi M, Filomenko R, Rébé C, Chevriaux A, Varin A, Derangère V, Gambert $P$, Lagrost $L$, Masson D: Knock-down of the oxysterol receptor LXRa impairs cholesterol efflux in human primary macrophages: lack of compensation by LXR $\beta$ activation. Biochem Pharmacol 2013, 86(1):122-129.

doi:10.1186/1471-2261-14-80

Cite this article as: Ma et al:: Cholesterol efflux is LXRa isoformdependent in human macrophages. BMC Cardiovascular Disorders $201414: 80$.

\section{Submit your next manuscript to BioMed Central and take full advantage of:}

- Convenient online submission

- Thorough peer review

- No space constraints or color figure charges

- Immediate publication on acceptance

- Inclusion in PubMed, CAS, Scopus and Google Scholar

- Research which is freely available for redistribution 